\title{
Dynamic Response Characteristics in Variable Stiffness Soft Inflatable Links
}

\author{
Ahmad Ali $^{1}$, Kaspar Althoefer ${ }^{1}$, Jelizaveta Konstantinova ${ }^{1}$ \\ ${ }^{1}$ The Centre for Advanced Robotics@ Queen Mary (ARQ), \\ Queen Mary University of London, UK \\ ahmad.w.ali@qmul.ac.uk
}

\begin{abstract}
In soft robotics, there is the fundamental need to develop devices that are flexible and can change stiffness in order to work safely in the vicinity of humans. Moreover, these structures must be rigid enough to withstand the force application and accuracy in motion. To solve these issues, previous research proposed to add a compliance element between motor and load - Series Elastic Actuators (SEAs). This approach benefits from improved force control and shock tolerance due to the elasticity introduced at joint level. However, series compliance at the joint level comes at the cost of inferior position controllability and additional mechanical complexity. In this research, we move the elastic compliance to the link, and evaluate the characteristics of variable stiffness soft inflatable links. The detailed investigation of the dynamic behaviour of inflatable link takes into consideration different internal pressures and applied loads. Our results demonstrate that the use of soft inflatable links leads to good weight lifting capability whilst preserving compliance which is beneficial for safety critical applications.
\end{abstract}

Keywords: Dynamic response, variable stiffness, inflatable soft link, humanrobot interaction

\section{Introduction}

In the last few years, there has been a growing interest in the development of soft robots. Soft robots feature compliance as the fundamental property in their structure. Traditional industrial robots are made of rigid components that have a remarkable performance in position controllability, high speed precision and large force output [1]. However, in other scenarios such as when it is required to perform tasks in a complex and nonstationary environment, as well as working in shared space with the human workers, rigid-bodies robots have disadvantages due to the lack of compliance, elasticity and safety. The introduction of elasticity in the robotic systems enables them to store and release energy at the time of need. This paradigm shift was introduced with the works of Hogan et al. on impedance [2], and of Pratt and Williamson on the concept of Series Elastic Actuators (SEAs) [3]. Their concept introduces an elastic element with constant stiffness between the gear and the actuator output. SEA minimizes the potential of injury in case of any contact with the environment and enables robotic manipulators to 
exhibit compliance in safe mode [4]. SEAs provide improvements for many realistic tasks such as shock tolerance, fidelity in force controllability and interaction with the environment. Recently some robotic devices, such as Sawyer or Baxter, introduce the use of inherently compliant actuators based on the concept of SEAs [5]. These commercially available robots are certified to work in the vicinity of human workers, hence stretching the frontiers of robotic systems. Usually, all SEAs are composed of a traditional electric motor in series with an elastic element (typically a spring) that, in turn, is connected to a robotic link. However, numerous expensive spring designs that are proposed in literature to realize the compact series elastic actuator result in high cost [6]. Moreover, this concept requires additional systems to modulate their stiffness in order to ensure shared autonomy factory environments with the human worker.

In recent years, research on soft robots has become very popular as such devices provide solutions to the issues of elasticity, compliance and safety. Therefore, there is a great potential for applications that require human-robot interaction in shared workspace. Soft robotic devices feature the inherit compliance over the whole structure rather than only at their joints as it is the case for SEAs. The natural compliance observed in biological organisms plays a central role in the exploration of novel soft robotic designs: the dexterity of natural limbs such as the trunk of the elephant [7], [8], the tentacle of the octopus [9] or the complex body motion of the snake [10], [11]. These biological systems are rarely composed of rigid mechanical components, but they generally make use of soft, elastic, and flexible materials in order to survive in complex unstructured environment [12], [13]. Therefore, soft robots are often made from soft, rubber or silicone-based polymers and fabrics that can enable complex body poses for whole-body manipulation and articulated movements in constrained environment [14].

However, so far too little attention has been paid to consider robot links that can change their stiffness to achieve compliance with the environment. The compliance in the links support the idea of autonomous sharing of the robot working space with the human workers. The inclusion of elasticity in the robotic link can replace the use of high-cost components relating to the SEA with a single inexpensive component: an inflatable, stiffness-controllable link made from low cost materials. Moreover, sensing the force at the link level has more significant advantages over the force sensing capability in a SEA which is at actuator level. Also, the robotic links with changing stiffness can respond promptly to any contact (either a perturbation or required interaction) at the environment level.

Current studies of variable stiffness soft robots are scarce with regards to soft inflatable links. Most collaborative robots are based on SEA implementations which add more complex mechanism near the actuators at joint level [15]. Our simple soft-link design provides a lightweight, low-cost solution with an opportunity to change stiffness at the link level. A continuum silicon-based soft manipulator was suggested to achieve change in stiffness via pneumatic pressure and tendons [16]. The experimental results showed that actuation mechanisms with tendons considerably increase load bearing capability. Although tendon-based solutions provide good control in position and orientation of manipulator, it lacks stiffness controllability. A variable stiffness link (VSL) concept was proposed with inherently elastic properties to work in a human-robot 
friendly environment [14]. These links are made of a number of chambers independently inflated in order to make them stiffer. However, these designs for VSLs does not investigate the dynamic behavior of inflatable link under different loading conditions.

Although the soft robotic approach has been seen many optimized designs for inflatable links, there are a number of limitations that researchers in this field are trying to overcome. Due to the deformability of soft robots, the modelling and control is more complex in comparison with their rigid counterparts [17]. This directly relates to what is widely considered as one of the core challenges of soft robotics: the stiffening of soft structures [18]. In the search for the right trade-off between desired compliance and exertable force, researchers explore numerous approaches to enable on-demand stiffness tuning of soft robots [19]. In addition, lightweight elastic links have challenging position control. Therefore, in this paper we investigate the dynamic behavior of soft and inflatable links under applied load and for different levels of pressure. The experiments reported here were designed to access the practicalities of using lightweight, lowcost inflatable link instead of rigid-link by taking into account the vibration analysis. In this article, we propose and investigate the dynamic behavior of a variable stiffness link (VSL) that is composed of an inner inflatable circular chamber and an outer layer of soft material.

This paper is organized as follows. First, we present the design and fabrication of VSL in Section II. The analytical modelling of the dynamic behavior for different pressure levels is presented in the Section III. The description of experimental setup and results is presented Section IV. Section V concludes our work, discussing the results obtained in the experiments and highlighting the achievements in developing soft inflatable links.

\section{Design and Fabrication of Variable Stiffness Links (VSLs)}

In this section, we describe the general principles of the design of VSL. In addition, the fabrication method of VSLs for the evaluation studies of the dynamic response is presented. Previously, our group has presented the design of the variable stiffness link in [15]. In that work, silicone is chosen as the base material/layer where a plastic mesh (polypropylene) with diamond shaped texture is incorporated. The composite material of silicon and plastic mesh create an inflatable chamber for pneumatic pressure that can change its stiffness based on the internal pneumatic pressure. In addition, non-stretchable fabric material is used as an outer skin to constrain the expansion of the soft silicone. The outer skin plays an important role in the stiffening of link as it puts the boundary conditions to avoid any ballooning of the internal elastic chamber and holds up the maximum expansion allowing to increase the stiffness.

In this work, we focus on understanding the dynamic responses of the VSL, and, hence, we employ a similar design concept when manufacturing our VSL prototypes. The proposed inflatable link is composed of two main structural elements: a circular 
inner chamber to encapsulate the pneumatic pressure and an outer skin layer. The component details of the soft inflatable link are depicted in Error! Reference source not found.. The inner chamber is made from a circular-shaped butyl rubber. This material is airtight and typically used as an inner bladder for sports equipment, such as balls. Several types of the outer material were tested for the outer skin, such as ripstop fabric, neoprene, leather and polythene membrane. The outer layer is wrapped around the inner chamber and fixed at the both ends of the link to ensure airtightness. Hard 3D printed circular caps from lightweight plastic are used to plug the ends of link. In addition, the ends caps are padded with rubber, sealed with a silicone glue and clamped with metal rings. The air supply tube is fixed at one end of the link. Further on, air is used to inflate the tube and to increase its stiffness.

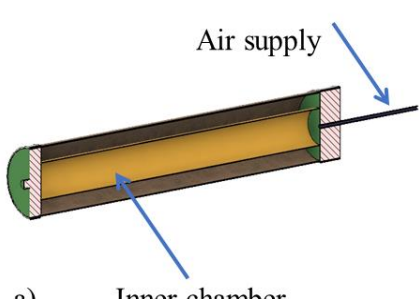

a) Inner chamber

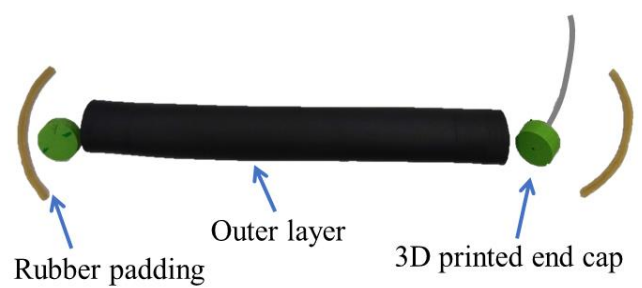

b)

Fig. 1. Design concept of the variable stiffness link: a) cross-section of the link showing the inner circular chamber; and b) exploded view of the VSL, showing the main components of the outer layer, and the 3D printed end cups with the rubber paddings for air tightness.

In order to fabricate the inflatable link, an inner tube with a diameter of $38 \mathrm{~mm}$ and a length of $400 \mathrm{~mm}$ is selected. The outer skin of the link is fabricated from a rectangular sheet of material, including ripstop fabric and suede leather. The width of the sheet is $70 \mathrm{~mm}$; which is shaped into a cylinder by wrapping the sheet around the circular endcaps. As a result, the fully inflated tube is $40 \mathrm{~mm}$ in diameter, and $400 \mathrm{~mm}$ in length.

a)
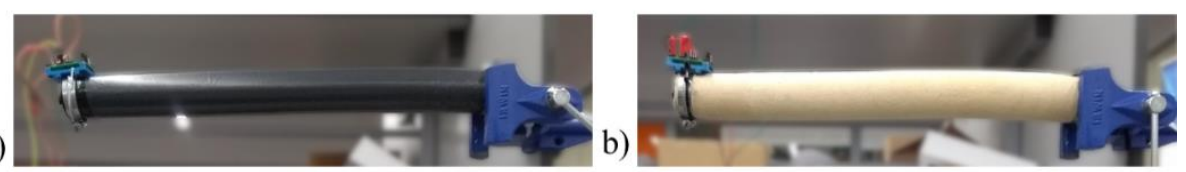

Fig. 2. Fabricated VSLs that are selected for experimental evaluation: a) ripstop fabric, and b) suede leather links.

\subsection{Evaluation of the material for the outer layer of VSL}

Further on, it is required to evaluate the feasibility of the different materials for use in the outer layer of the link. Four links were fabricated and inflated to the maximum pressure of 43 psi. Further on, we discuss the mechanical properties observed for the selected materials. Neoprene layer creates a flexible, lightweight and air tight link. However, its highly elastic behavior leads to the ballooning of internal tube. In addition, 
the rapture of the link structure is observed for a pressure of about $20 \mathrm{psi}$. The polythene membrane is selected as a possible material for the outer skin of the inflatable link due its air tightness, and also because it is lightweight and low-cost. The link exhibits a considerable amount of stiffness when the pressure is applied. However, when the pressure exceeds $18 \mathrm{psi}$, wrinkling of the material is observed and there is the danger that the link material can rupture. Finally, both soft suede leather and ripstop fabric links were successfully tested and selected as a materials for the outer layer (Error! Reference source not found.). The results of the dynamic response testing are presented in Section 4.

\section{$3 \quad$ Analytical Modelling}

The dynamic behavior of variable stiffness soft inflatable links needs to be investigated in order to create robust control strategies. Therefore, one of the core issues in the modelling of soft inflatable links is the mathematical framework that can predict their physical behavior. During the operation of the link, the inner circular chamber is equally inflated along the length of link. The air inside the tube is acting as a compressible fluid that can change the working pressure of the VSL. The impact of this working pressure creates a considerable amount of force on the surface of circular chamber, inflating the robotic link. The VSL is a continuous circular tube, and when it is inflated it can be approximated as a continuous elastic beam. The Pascal's law states that if there is an increase in pressure at any point in a confined fluid, an equal increase in force at every point in the container is realized. According to that, the inner pressure of the VSEL remains the same along the length of the link. Further on, the use of different pneumatic pressure can be used in changing and controlling the stiffness of the inflatable link.

The stiffness of the VSL is a result of the combined effects of the inertia and elastic properties of materials used for inner chamber and outer skin. The inertia of the soft inflatable link is compared to the change in the density of the air particles when the pneumatic pressure inside the inner chamber is changed. This can be expressed by the ideal gas equation (1) [20]:

$$
P V=m R T,
$$

where $\mathrm{P}$ is the maximum pressure inside the tube; $\mathrm{V}$ is the volume of the link at maximum inflation; $\mathrm{R}$ is the gas constant; $\mathrm{m}$ is the mass of air particles; and $\mathrm{T}$ is the temperature of the air particles. At the beginning of the operation, the pneumatic pressure is used to inflate the VSL (Error! Reference source not found.). Once the inner tube reaches its maximum expansion, i.e., the volume of the link becomes constant, the stiffness of the link increases. Hence, increasing the pneumatic pressure means that larger number of air mass is being accumulated inside the tube leading to higher stiffness of the VSL. The accumulated air mass (inertia) of the link becomes a function of supply pressure because all other physical quantities $V, T, R$, are constant except $P$ and $m$. This shows that the stiffness is a function of number of air particles that lead to an increase in the inertia of the inflatable link. This variation in the inertial property makes the inflatable links suitable for applications that require stiffness controllability. 
An inflatable link at the maximum pressure can be modelled as a horizontal cantilever beam fixed at one end. When the dynamic load is applied, the link goes into vibrations at its natural frequency. If the inner tube inside the link is packed densely with air mass through an increased pneumatic pressure, then the flexural rigidity is changed. This change in flexural properties of the inflatable link causes the natural frequency of the vibration to shift. When the inflatable link goes into free vibrations after the impact of point loading at the free end of the link, the equation of motion that describes its dynamic response assuming equally distributed gas molecules inside the chamber [21], is as follows:

$$
\frac{d^{2}}{d x^{2}}\left\{E I(x) \frac{d^{2} \mathrm{Z}(\mathrm{x})}{d x^{2}}\right\}=\omega^{2} m(x) \mathrm{Z}(\mathrm{x}),
$$

where, $E$ is the modulus of elasticity of the beam material, $I$ is the moment of inertia of the circular section of inflatable, $Z(x)$ is the displacement in $z$ direction at distance $x$ from the fixed end of the link, $\omega$ is the circular natural frequency, $m$ is the mass per unit length: $m=\rho \mathrm{A}(\mathrm{x})$. In this equation, $\rho$ is the mass density of air particles, $x$ is the distance measured from the fixed end of the link. The following boundary conditions can be considered in case the load is applied in the normal direction:

$$
\begin{gathered}
x=0, Z(x)=0, \frac{\partial Z(x)}{\partial(x)}=0, \\
x=1, \quad \frac{\partial^{2} Z(x)}{\partial\left(x^{2}\right)}=0, \quad \frac{\partial^{3} Z(x)}{\partial\left(x^{3}\right)}=0 .
\end{gathered}
$$

Each vibrating system (in our case - the inflatable link) exhibits its own natural frequency when it is deflected by an external forcing agent. The natural frequency of the system is defined as:

$$
f=\frac{k_{n}^{2}}{2 \pi L} \sqrt{\frac{E I}{\rho A}} .
$$

Equation (5) demonstrates that a vibrating system gradually starts getting higher natural frequency if the Young's modulus is increased, e.g. by pneumatic pressure.

\section{Evaluation of the dynamic response for VSL}

\subsection{Experimental setup}

The main focus of the experiments is to observe the dynamic response of the link when it is subject to impact loading. Moreover, employing the feature of variable stiffness, we are testing the impact of the internal pneumatic pressure. Based on the results obtained in Section 2.1, the dynamic responses of both suede leather and ripstop fabric links are tested. The experimental setting is shown in Fig. 3. . The link is positioned 
horizontally, and the proximal end connected to the air supply is tightly fixed to avoid any displacement at the base. During the experiments, the stiffness of the VSL was increased from 6 psi to 24 psi at steps of 3 psi. Further on, the load was applied to the link for each pressure level. The load was increased from $1 \mathrm{~N}$ to $5 \mathrm{~N}$ at steps of $1 \mathrm{~N}$. The load was attached via small thread at the suspended end of the VSL and, then, cut to observe the dynamic response of the link. Each experiment was repeated 3 times.

The dynamic response of the link is measured using an LIS344ALH 3-axis linear capacitive accelerometer. The acceleration measurement is recorded using a PicoScope 2000 oscilloscope with a measurement bandwidth of $25 \mathrm{MHz}$. The pressure control for the stiffness variability is implemented with SMC ITV electro-pneumatic pressure regulator. The control of the regulator was implemented using an Arduino Mega SDK board.
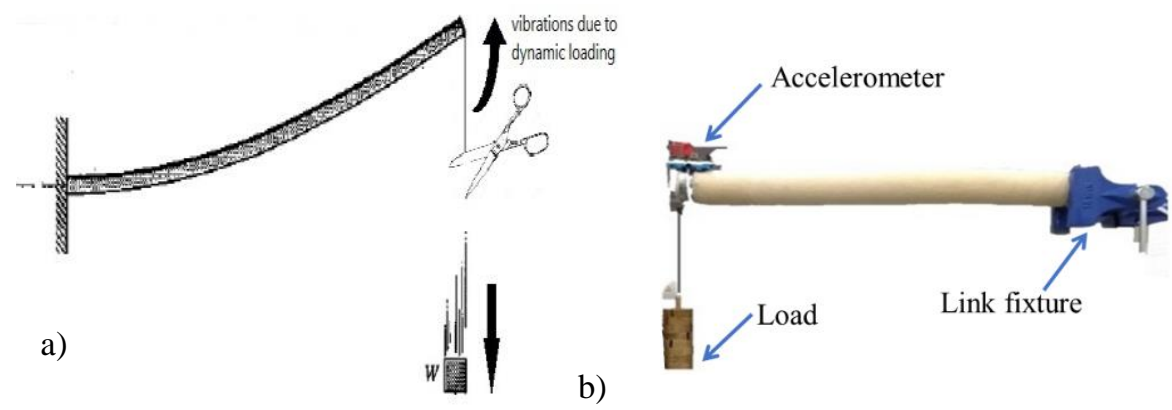

Fig. 3. Evaluation of the dynamic response of VSL: a) sketch of the experimental procedure, and b) suede leather link with the maximum weigh of $5 \mathrm{~N}$ applied.

\subsection{Analysis of the Experimental Results}

This section presents the analysis and evaluation of the experimental study presented above. The aim of the study is to understand the dynamic characteristics of the VSL during the application of load. In addition, the effect of the pressure level inside VSL is evaluated. The data was processed using MATLAB 2018b, and the statistical analysis was performed using R-statistical software. The significance level or the probability of rejecting the null hypothesis is defined as 0.05 , with a confidence interval of $95 \%$.

For the experimental evaluation, displacement and velocity data was obtained from the recorded acceleration using cumulative trapezoidal numerical integration. The sample response of the displacement pattern for the leather link during the application of a fixed load is shown in Fig. 4. It can be observed that the link displacement is following a vibration pattern that is settling down after a period of time.

To understand whether the settling time is changing for different pressures and applied load, the settling time was analyzed for both leather and fabric links and a different pressure. The mean settling time for the leather link is $1.164 \mathrm{~s}$, with a standard deviation of $0.211 \mathrm{~s}(18 \%)$. In addition, similar values can be observed for the link made from fabric - we observe a mean settling time of $1.484 \mathrm{~s}$, and a standard deviation of 0.275 $\mathrm{s}(18.5 \%)$. According to nonparametric Kruskal Wallis test, there is no relationship of 
settling time with pressure ( $p=0.153)$ and applied load $(p=0.472)$ for leather. However, it was observed that the settling time of the fabric link depends on the applied weight ( $\mathrm{p}<0.005)$. It can be observed that the settling time decreases for a load of $3 \mathrm{~N}$, Fig. 5. The applied pressure does not impact the settling time of the vibrations for the fabric link $(p=0.904)$, similarly as it is for the leather link.
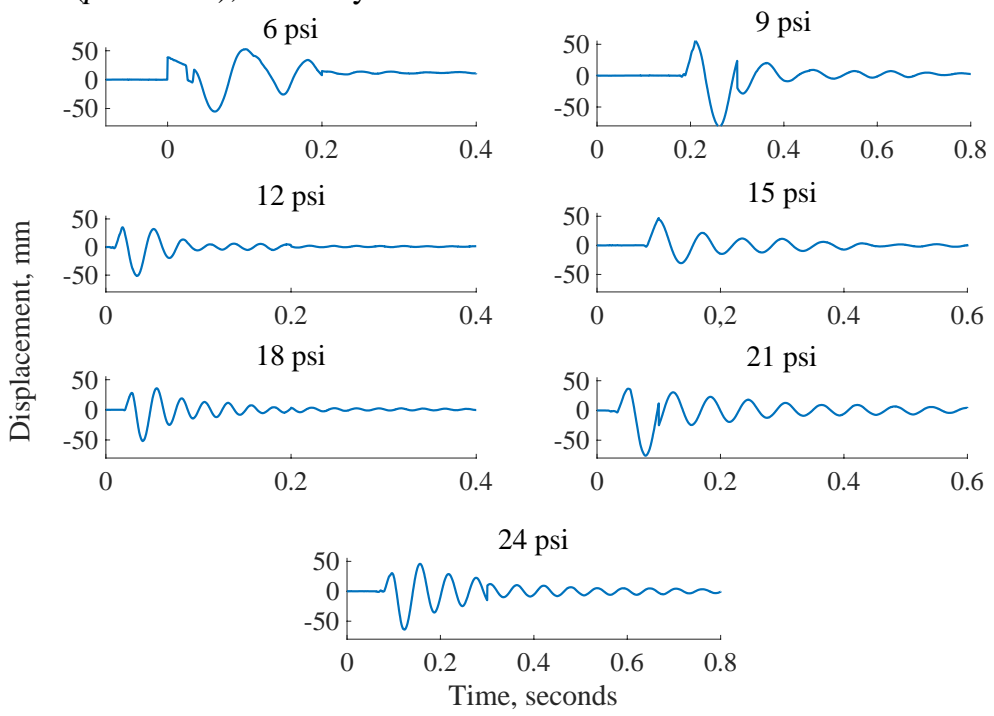

Fig. 4. Sample response for leather link for different internal pressures and an applied load of 3 $\mathrm{N}$.
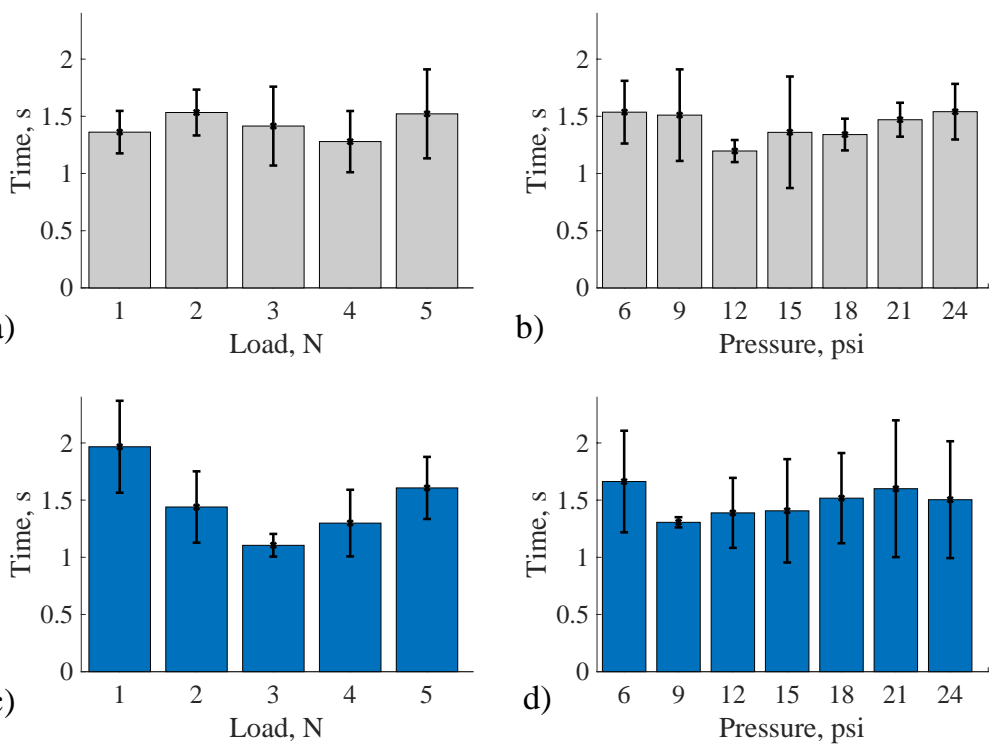
Fig. 5. Settling time for a) leather link across different loads; b) leather link for different pressure values; c) fabric link across different loads; d) fabric link for different pressure values.

The resultant frequency of the response for both leather and fabric links is evaluated, as one of the important parameters of the dynamic analysis (Fig. 6). According to statistical analysis, it was noted that there is a significant relationship with the applied pressure for both leather and fabric links $(p<0.005)$. As it is demonstrated in Fig. 6, the response frequency is increasing together with the internal pressure. There is no relationship between frequency and applied load; with $p=0.158$ and $p=0.056$ for leather and fabric links respectively. It was also noted that the range of the response is similar for both leather and fabric links, ranging from $4.5 \mathrm{~Hz}$ to $17 \mathrm{~Hz}$.
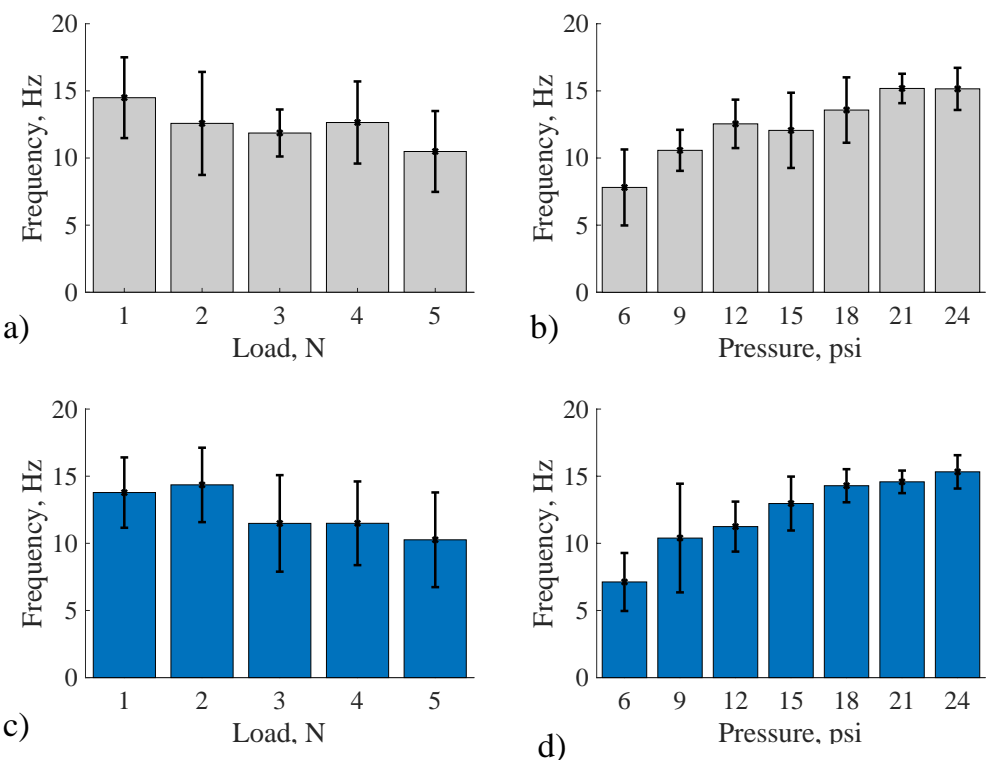

Fig. 6. Response frequency for a) leather link across different loads; b) leather link for different pressure levels; c) fabric link across different loads; d) fabric link for different pressure levels.

In order to evaluate the vibration response of the inflatable links, similar experiments were performed with hollow links made from rigid materials, including plastic and steel. Two rigid links comparable in dimensions with the inflatable links were selected to perform a comparative study (Fig. 7). The first link is a hollow steel tube with a wall thickness of $3 \mathrm{~mm}$. The second link is made from rigid and lightweight PVC (polyvinyl chloride) with a wall thickness of $2 \mathrm{~mm}$. The length and diameter of both links are 400 $\mathrm{mm}$ and $40 \mathrm{~mm}$, respectively. Force stimuli ranging for $1 \mathrm{~N}$ to $20 \mathrm{~N}$ were applied to these links, as shown in Fig. 8. It was found that the average resultant frequency of the response for steel and PVC links is $84.5 \mathrm{~Hz}$ with a standard deviation of $6.9 \mathrm{~Hz}$ and $79.8 \mathrm{~Hz}$ with a standard deviation of $3.6 \mathrm{~Hz}$, respectively. It can be seen that the response frequency of rigid links is considerably higher than the response frequency of the inflatable ones. 

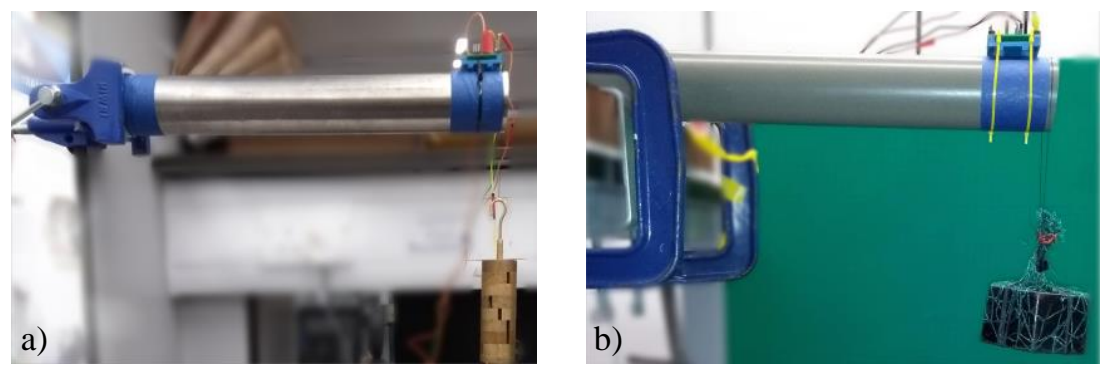

Fig. 7. Hard links for comparative study: a) steel tube, and b) PVC link.

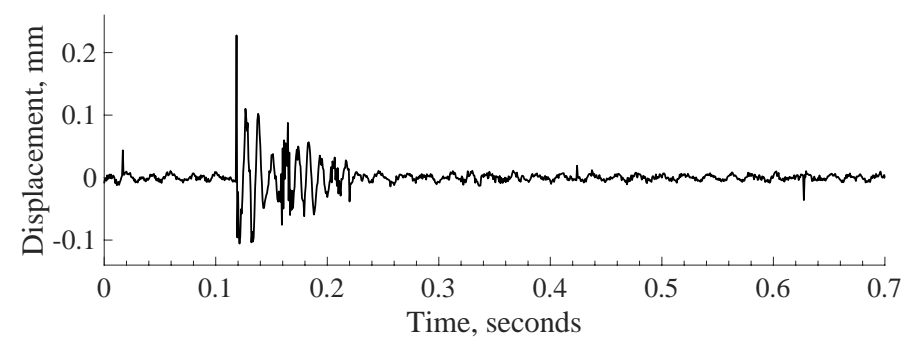

Fig. 8. Sample displacement response of the steel link; the applied force in the shown case is 20 $\mathrm{N}$.

\section{Conclusions}

In this paper we propose the initial evaluation of the dynamic response for variable stiffness links (VSLs). This knowledge can shed light on the dynamic behavior of soft and inflatable links. VSLs are capable of achieving stiffness modulation by varying the applied pneumatic pressure. The links benefit from the fact that they are lightweight and made of low-cost materials. It was found that the most suitable outer layer of the VSL is soft fabric or leather. However, the dynamic response characteristics, such as settling time can be influenced by the material of the outer layer. In future work, it is planned to perform additional dynamic studies and to develop control strategies, including those based on neuro-fuzzy paradigms, for the proposed variable stiffness link concept.

\section{References}

1. B. Siciliano and O. Khatib, Handbook on Robotics. Springer, 2014.

2. N. Hogan, "Impedance Control: An Approach to Manipulation: Part III-Applications," J. Dyn. Syst. Meas. Control, 1985.

3. G. Pratt and M. Williamson, "Series Elastic Actuators," Proc. 1995 IEEE/RSJ Int. Conf. Intell. Robot. Syst. Hum. Robot Interact. Coop. Robot., vol. 1, pp. 399-406, 1995. 
4. S. Wolf et al., "Variable Stiffness Actuators: Review on Design and Components," IEEE/ASME Transactions on Mechatronics. pp. 2418-2430, 2016.

5. C. Yang, J. Luo, Y. Pan, Z. Liu, and C. Y. Su, "Personalized Variable Gain Control With Tremor Attenuation for Robot Teleoperation," IEEE Transactions on Systems, Man, and Cybernetics: Systems, 2017.

6. J. E. Colgate and G. G. Schenkel, "Passivity of a class of sampled-data systems: Application to haptic interfaces," J. Robot. Syst., 1997.

7. J. Yang, E. P. Pitarch, J. Potratz, S. Beck, and K. Abdel-Malek, "Synthesis and analysis of a flexible elephant trunk robot," Adv. Robot., 2006.

8. R. Cieślak and A. Morecki, "Elephant trunk type elastic manipulator - a tool for bulk and liquid materials transportation," Robotica, vol. 17, no. 1. pp. 11-16, 1999.

9. C. Laschi, B. Mazzolai, V. Mattoli, M. Cianchetti, and P. Dario, "Design of a biomimetic robotic octopus arm.," Bioinspir. Biomim., vol. 4, no. 1, p. 015006, 2009.

10. S. Hirose, "Biologically Inspired Robots: Snake-Like Locomotors and Manipulators," Appl. Mech. Rev., vol. 48, no. 3, pp. B27-B27, 1995.

11. D. Rollinson et al., "Design and architecture of a series elastic snake robot," in 2014 IEEE/RSJ International Conference on Intelligent Robots and Systems, IROS 2014, September 14, 2014 - September 18, 2014, 2014, pp. 4630-4636.

12. C. Majidi, "Soft Robotics: A Perspective-Current Trends and Prospects for the Future," Soft Robot., 2014.

13. D. Rus and M. T. Tolley, "Design, fabrication and control of soft robots," Nature, 2015.

14. A. Stilli, L. Grattarola, H. Feldmann, H. A. Wurdemann, and K. Althoefer, "Variable Stiffness Link (VSL): Toward inherently safe robotic manipulators," in Proceedings - IEEE International Conference on Robotics and Automation, 2017.

15. A. Stilli, H. A. Wurdemann, and K. Althoefer, "A Novel Concept for Safe, StiffnessControllable Robot Links," Soft Robot., 2017.

16. A. Shiva et al., "Tendon-Based Stiffening for a Pneumatically Actuated Soft Manipulator," IEEE Robot. Autom. Lett., vol. 1, no. 2, pp. 632-637, 2016.

17. H. Lipson, "Challenges and opportunities for design, simulation, and fabrication of soft robots," Soft Robot., vol. 1, no. 1, pp. 21-27, 2014.

18. M. Manti, V. Cacucciolo, and M. Cianchetti, "Stiffening in soft robotics: A review of the state of the art," IEEE Robot. Autom. Mag., 2016.

19. L. Blanc, A. Delchambre, and P. Lambert, "Flexible Medical Devices: Review of Controllable Stiffness Solutions," Actuators, 2017.

20. P. Atkin and J. Paula, Physical chemistry. 2006.

21. L. Meirovitch, Anayltical Methods in Vibrations. 1967. 